\title{
Effect of core electrical conductivity on core surface flow models
}

\author{
Masaki Matsushima* (i)
}

\begin{abstract}
The electrical conductivity of the Earth's core is an important physical parameter that controls the core dynamics and the thermal evolution of the Earth. In this study, the effect of core electrical conductivity on core surface flow models is investigated. Core surface flow is derived from a geomagnetic field model on the presumption that a viscous boundary layer forms at the core-mantle boundary. Inside the boundary layer, where the viscous force plays an important role in force balance, temporal variations of the magnetic field are caused by magnetic diffusion as well as motional induction. Below the boundary layer, where core flow is assumed to be in tangentially geostrophic balance or tangentially magnetostrophic balance, contributions of magnetic diffusion to temporal variation of the magnetic field are neglected. Under the constraint that the core flow is tangentially geostrophic beneath the boundary layer, the core electrical conductivity in the range from $10^{5} \mathrm{~S} \mathrm{~m}^{-1}$ to $10^{7} \mathrm{~S} \mathrm{~m}^{-1}$ has less significant effect on the core flow. Under the constraint that the core flow is tangentially magnetostrophic beneath the boundary layer, the influence of electrical conductivity on the core flow models can be clearly recognized; the magnitude of the mean toroidal flow does not increase or decrease, but that of the mean poloidal flow increases with an increase in core electrical conductivity. This difference arises from the Lorentz force, which can be stronger than the Coriolis force, for higher electrical conductivity, since the Lorentz force is proportional to the electrical conductivity. In other words, the Elsasser number, which represents the ratio of the Lorentz force to the Coriolis force, has an influence on the difference. The result implies that the ratio of toroidal to poloidal flow magnitudes has been changing in accordance with secular changes of rotation rate of the Earth and of core electrical conductivity due to a decrease in core temperature throughout the thermal evolution of the Earth.
\end{abstract}

Keywords: Core electrical conductivity, Core surface flow, Tangentially geostrophic flow, Tangentially magnetostrophic flow, Elsasser number

\section{Introduction}

The intrinsic magnetic field of the Earth is generated by dynamo action due to electromagnetic fluid motion in the outer core, the main components of which are iron and nickel. It is essential that the motional induction overcomes the magnetic diffusion through Ohmic dissipation to maintain the geomagnetic field. The magnetic diffusivity, $\eta$, is given as $\eta=\left(\mu_{0} \sigma\right)^{-1}$, where $\mu_{0}$ and $\sigma$ are the magnetic permeability of a vacuum and

\footnotetext{
*Correspondence: masaki.matsushima@eps.sci.titech.ac.jp Department of Earth and Planetary Sciences, Tokyo Institute of Technology, 2-12-1 Ookayama, Meguro, Tokyo 152-8551, Japan
}

core electrical conductivity, respectively. Therefore, higher electrical conductivity of core fluid is preferred for easier generation of the geomagnetic field against the magnetic diffusion. Higher electrical conductivity means higher thermal conductivity, $k$, of the metallic core, as both electrical and thermal conduction are dominated by the electron contribution. This can also be found from the Wiedemann-Franz law, $k=L_{o} T \sigma$, where $L_{o}=2.44 \times 10^{-8} \mathrm{~W} \Omega \mathrm{K}^{-2}$ and $T$ are the Lorentz number and temperature, respectively. Excessive thermal conductivity indicates that no thermal convection in the core is required to release heat from the core to the mantle (e.g., Pozzo et al. 2012). This does not necessarily mean 
that another type of convection, such as compositional convection, does not occur. In reality, the Earth has possessed its intrinsic magnetic field generated by the geodynamo since around $3.45 \mathrm{Ga}$ (Tarduno et al. 2010).

An assessment of the convective motions generating the geomagnetic field has been advanced by numerical simulations of the geodynamo. Noticeable columnar convective structures parallel to the rotational axis of the Earth are found to explain the generation mechanism of the axial dipole magnetic field (e.g., Kageyama and Sato 1997; Olson et al. 1999). Furthermore, numerical geodynamo models have succeeded in explaining certain properties of the geomagnetic field, such as the dominance of the dipole field, and secular variations including polarity reversals (e.g., Christensen and Wicht 2015). However, the convective motions produced by numerical simulations do not necessarily show proper core dynamics, mainly because the parameters adopted in numerical simulations are far from the real ones.

Useful information on core dynamics, features of the core-mantle boundary (CMB), and core-mantle coupling, for example, can be provided by realistic fluid motion in the core of the Earth. This core fluid motion can be estimated from spatial and temporal distributions of the geomagnetic field (e.g., Holme 2015). Most core surface flow models rely on the frozen-flux approximation (Roberts and Scott 1965), in which the magnetic diffusion is neglected. However, a viscous boundary layer is present at the $\mathrm{CMB}$, where the magnetic diffusion plays an important role in secular variations of the geomagnetic field (Takahashi et al. 2001). Therefore, a new approach to estimate fluid flow near the core surface has been devised by Matsushima (2015). In this approach, the magnetic diffusion is explicitly incorporated within the viscous boundary layer at the $\mathrm{CMB}$, whereas it is neglected below the boundary layer. Moreover, the fluid flow below the boundary layer is presumed to be tangentially geostrophic. A core flow model inside and below the viscous boundary layer at the $\mathrm{CMB}$ can then be derived from a geomagnetic field model.

In this method, core electrical conductivity can play a role in estimating core surface flows. The temporal variations in the radial component of the magnetic field, $B_{r}$, at the CMB are caused only by magnetic diffusion because of the no-slip condition for core flows there. The second partial derivative of $B_{r}$ with respect to the radius is thus related to core electrical conductivity. This suggests that core electrical conductivity can be influential in inferring $B_{r}$ inside the core. It should be noted that core electrical conductivity can also be crucial in the estimation of core flow, because the Lorentz force in the equation of motion depends on the electrical current density proportional to electrical conductivity.
Hence, in this paper, the effects of core electrical conductivity on core surface flow models are investigated for various values of core electrical conductivity that are still controversial (e.g., Ohta et al. 2016; Konôpková et al. 2016; Xu et al. 2018). First, the method of Matsushima (2015), in which core flow is presumed to be tangentially geostrophic below a viscous boundary layer at the core surface, is recalled. The method is then developed to include not only the effect of the Coriolis force, but also that of the Lorentz force. To investigate the effect of core electrical conductivity, this value is varied as a parameter. These results are discussed and summarized.

\section{Theory}

Matsushima (2015) gave a method for estimating fluid flow near the core surface with magnetic diffusion in a viscous boundary layer. Here this theory is recalled in brief, and the method is extended to include not only the effect of the Coriolis force for a tangentially geostrophic flow, but also that of the Lorentz force for a tangentially magnetostrophic flow. The radial component, which is denoted by subscript $r$, of the induction equation is given as

$$
\begin{aligned}
\dot{B}_{r i}= & \left\{-\left(\boldsymbol{V}_{i} \cdot \nabla\right) B_{r i}+\left(\boldsymbol{B}_{i} \cdot \nabla\right) V_{r i}\right\}\left(\delta_{i 1}+\delta_{i 2}\right) \\
& +\frac{\eta}{r_{i}} \nabla^{2}\left(r_{i} B_{r i}\right)\left(\delta_{i 0}+\delta_{i 1}\right),
\end{aligned}
$$

where $\boldsymbol{B}$ is the magnetic field, $\boldsymbol{V}$ the velocity of incompressible core fluid, and $\delta_{i j}$ the Kronecker delta. A dot denotes partial differentiation with respect to time, $t$. The other subscript, $i$, indicates the depth to be considered: $i=0$ at the CMB (assumed to be a spherical surface with radius $\left.r=r_{0}=3480 \mathrm{~km}\right), i=1$ inside the boundary layer $\left(r=r_{1}=r_{0}-\xi_{1}\right)$ at a depth of $\xi_{1}$ from the CMB, and $i=2$ below the boundary layer $\left(r=r_{2}=r_{0}-\xi_{2}\right)$ at a depth of $\xi_{2}$. For $i=0$, where the core flow relative to a reference frame rotating with the mantle must vanish under the no-slip condition, the first and second terms of the right-hand side of Eq. (1) must also vanish, and only the third term, or magnetic diffusion term, remains; that is, temporal variations of the magnetic field at the CMB arise from the magnetic diffusion only. For $i=1$, all three right-hand-side terms contribute to temporal variation of the geomagnetic field. For $i=2$, the magnetic diffusion term is presupposed to be negligible, although the thickness of the magnetic boundary layer would be thicker than that of the viscous boundary layer (e.g., Chulliat and Olsen 2010), because contribution of the motional induction to temporal variations in the magnetic field is likely to be much larger than that of the magnetic diffusion, as in the frozen-flux approximation (e.g., Holme 2015). 
Core flow is assumed to be tangentially geostrophic below the boundary layer, while the viscous force is presumed to play an important role inside the boundary layer. Therefore, core flow $\boldsymbol{V}_{1}$ and $\boldsymbol{V}_{2}$ should satisfy Eqs. (2a) and (2b), respectively:

$$
\begin{aligned}
& \hat{\boldsymbol{r}} \cdot \nabla \times\left(-2 \boldsymbol{\Omega} \times \boldsymbol{V}_{1}+v_{\text {edd }} \nabla^{2} \boldsymbol{V}_{1}\right)=0, \\
& \hat{\boldsymbol{r}} \cdot \nabla \times\left(-2 \boldsymbol{\Omega} \times \boldsymbol{V}_{2}\right)=0,
\end{aligned}
$$

where $\boldsymbol{\Omega}$ denotes the angular velocity vector of the mantle, $\hat{r}$ the radial unit vector, and $v_{\text {edd }}$ the eddy kinematic viscosity. The typical length scale parallel to the boundary layer is likely to be much larger than the thickness of the boundary layer. The horizontal flow, $V_{H}$, near the core surface is then expressed as in classical Ekman layer theory (e.g., Pedlosky 1987),

$$
\begin{aligned}
\boldsymbol{V}_{H}= & \overline{\boldsymbol{V}}_{H}\left\{1-\exp \left(-\frac{\xi}{\delta_{E}}\right) \cos \left(\frac{\xi}{\delta_{E}}\right)\right\} \\
& +(\operatorname{sgn} \cos \theta) \hat{\boldsymbol{r}} \times \overline{\boldsymbol{V}}_{H} \exp \left(-\frac{\xi}{\delta_{E}}\right) \sin \left(\frac{\xi}{\delta_{E}}\right),
\end{aligned}
$$

where sgn is the signum function, $\delta_{E}=\left(v_{\text {edd }} / \Omega|\cos \theta|\right)^{1 / 2}$ with $\Omega=|\boldsymbol{\Omega}|=7.29 \times 10^{-5} \mathrm{rad} \mathrm{s}^{-1}$, and $\theta$ is the colatitude in the spherical coordinates $(r, \theta, \phi)$. The tangentially geostrophic flow, $\overline{\boldsymbol{V}}_{H}$, significantly below the viscous boundary layer should satisfy Eq. (4) obtained from Eq. (2b):

$\nabla_{H} \cdot\left(\cos \theta \bar{V}_{H}\right)=0$,

where $\nabla_{H}$ is the horizontal gradient, and $\bar{V}_{r}$ is significantly smaller than $\left|\overline{\boldsymbol{V}}_{H}\right|$ and can be neglected. For the case of tangentially geostrophic flow, core electrical conductivity $\sigma$ has an effect on the magnetic diffusion alone, which leads to second partial derivatives of $B_{r}$ at $r=r_{0}$ with respect to the radius.

To examine the effect of $\sigma$ on a core flow model, core flow is next assumed to be tangentially magnetostrophic below the boundary layer, which is an Ekman-Hartmann layer in this case. Therefore, core flow $V_{1}$ and $V_{2}$ should satisfy Eqs. (5a) and (5b), respectively:

$$
\begin{aligned}
& \hat{\boldsymbol{r}} \cdot \nabla \times\left(-2 \boldsymbol{\Omega} \times \boldsymbol{V}_{1}+\rho^{-1} \boldsymbol{J}_{1} \times \boldsymbol{B}_{1}+v \nabla^{2} \boldsymbol{V}_{1}\right)=0, \\
& \hat{\boldsymbol{r}} \cdot \nabla \times\left(-2 \boldsymbol{\Omega} \times \boldsymbol{V}_{2}+\rho^{-1} \boldsymbol{J}_{2} \times \boldsymbol{B}_{2}\right)=0,
\end{aligned}
$$

where $\rho$ and $\boldsymbol{J}$ denote the mass density of the core fluid and the electric current density, respectively. In this study, as mentioned in Appendix, contribution of the electric field to the current density is ignored (e.g., Shimizu 2006), and $J_{r}$ is likely to be much smaller than $\left|J_{H}\right|$ near the mantle, which is assumed to be an electrical insulator (Benton and Muth 1979). The horizontal component of current density is then given as

$$
\boldsymbol{J}_{H}=\sigma(\boldsymbol{V} \times \boldsymbol{B})_{H} \approx \sigma B_{r} \boldsymbol{V}_{H} \times \hat{\boldsymbol{r}} .
$$

The horizontal flow, $\boldsymbol{V}_{H}$, near the core surface is expressed as

$$
\begin{aligned}
\boldsymbol{V}_{H}= & \overline{\boldsymbol{V}}_{H}\left\{1-\exp \left(-\frac{\xi}{\delta_{E H}^{+}}\right) \cos \left(\frac{\xi}{\delta_{E H}^{-}}\right)\right\} \\
& +(\operatorname{sgn} \cos \theta) \hat{\boldsymbol{r}} \times \overline{\boldsymbol{V}}_{H} \exp \left(-\frac{\xi}{\delta_{E H}^{+}}\right) \sin \left(\frac{\xi}{\delta_{E H}^{-}}\right),
\end{aligned}
$$

and the tangentially magnetostrophic flow, $\overline{\boldsymbol{V}}_{H}$, significantly below the viscous boundary layer satisfies

$$
\nabla_{H} \cdot\left(2 \Omega \cos \theta \overline{\boldsymbol{V}}_{H}+\rho^{-1} \sigma B_{r 2}^{2} \overline{\boldsymbol{V}}_{H} \times \hat{\boldsymbol{r}}\right)=0 .
$$

Here, $\delta_{E H}^{+}$and $\delta_{E H}^{-}$are given by

$$
\delta_{E H}^{ \pm}=\frac{\delta_{E}}{\left\{\left(1+\Lambda^{2} / 4\right)^{1 / 2} \pm \Lambda / 2\right\}^{1 / 2}}
$$

(double sign correspondence), and

$$
\Lambda=\frac{\sigma B_{r}^{2}}{\rho \Omega|\cos \theta|}
$$

is the Elsasser number. For the case of tangentially magnetostrophic flow, core electrical conductivity $\sigma$ has an effect not only on the magnetic diffusion, but also on the magnetostrophy through the Lorentz force.

The horizontal geostrophic (or magnetostrophic) velocity can be expressed in terms of poloidal and toroidal constituents as

$$
\bar{V}_{H}=r \nabla_{H} \bar{U}+\nabla \times(r \bar{W}),
$$

$\bar{U}(\theta, \phi, t)=\sum_{l=1}^{L} \sum_{m=0}^{l}\left\{\bar{U}_{l}^{m c}(t) \cos m \phi+\bar{U}_{l}^{m s}(t) \sin m \phi\right\} P_{l}^{m}(\cos \theta)$,

$\bar{W}(\theta, \phi, t)=\sum_{l=1}^{L} \sum_{m=0}^{l}\left\{\bar{W}_{l}^{m c}(t) \cos m \phi+\bar{W}_{l}^{m s}(t) \sin m \phi\right\} P_{l}^{m}(\cos \theta)$,

where $P_{l}^{m}$ is a Schmidt-normalized associated Legendre function of degree $l$ and order $m, L$ is the truncation level, $r$ is a position vector, and $\bar{U}$ and $\bar{W}$ are poloidal and toroidal scalar functions, respectively. 


\section{Method}

To obtain a core surface flow model for a long duration, a geomagnetic field model, COV-OBS.x1 (Gillet et al. 2015), ranging from 1840 to 2015, is adopted. It should be noted that the period 1840-1880 in the COV-OBS. x1 model may contain a problem (Metman et al. 2018). Therefore, means and standard deviations are calculated in the range from 1880 to 2015 in this study. The magnetic field at the CMB is derived through downward continuation of a geomagnetic potential field by assuming the mantle to be an electrical insulator as

$$
\begin{aligned}
& B_{r 0}(\theta, \phi, t)=\sum_{l=1}^{L}(l+1)\left(\frac{r_{e}}{r_{o}}\right)^{l+2} \\
& \sum_{m=0}^{l}\left\{g_{l}^{m}(t) \cos m \phi+h_{l}^{m}(t) \sin m \phi\right\} P_{l}^{m}(\cos \theta),
\end{aligned}
$$

where $r_{e}=6371 \mathrm{~km}$ is the mean radius of the Earth, and $g_{l}^{m}$ and $h_{l}^{m}$ are the Gauss coefficients given by the COVOBS.x1 model. The truncation level of spherical harmonic coefficients is set at degree $L=14$.

The radial component of the geomagnetic field shallow inside the core, $B_{r 1}$ and $B_{r 2}$, can be estimated using a Taylor expansion at $r=r_{0}$ as

$$
B_{r i}=B_{r 0}-\xi_{i} \frac{\partial B_{r 0}}{\partial r}+\frac{\xi_{i}^{2}}{2} \frac{\partial^{2} B_{r 0}}{\partial r^{2}}(i=1,2),
$$

where the second term of the right-hand side of Eq. (14) can be obtained from $\nabla \cdot \boldsymbol{B}=0$, and the third term from Eq. (1) at $r=r_{0}$ (Matsushima 2015). Their time derivatives, $\dot{B}_{r 1}$ and $\dot{B}_{r 2}$, can be derived from $\nabla \cdot \dot{\boldsymbol{B}}=0$ and $\ddot{B}_{r 0}=\left(\eta / r_{0}\right) \nabla^{2}\left(r_{0} \dot{B}_{r 0}\right)$. Then, Eq. (1) at $r=r_{1}$ and $r=r_{2}$ with a constraint Eq. (4) or Eq. (8) are solved in physical space, at grid points $\left(\theta_{k}, \phi_{k}\right)$ as

$$
\left(\begin{array}{c}
\boldsymbol{d}_{1} \\
\boldsymbol{d}_{2} \\
\mathbf{0}
\end{array}\right)=\left(\begin{array}{c}
\mathbf{A}_{1} \\
\mathbf{A}_{2} \\
\alpha \mathbf{A}_{g}
\end{array}\right) \cdot g
$$

where $\boldsymbol{d}_{1}$ and $\boldsymbol{d}_{2}$ contain $\dot{B}_{r 1}\left(\theta_{k}, \phi_{k}\right)$ and $\dot{B}_{r 2}\left(\theta_{k}, \phi_{k}\right)$, respectively; $\mathbf{A}_{1}$ and $\mathbf{A}_{2}$ are matrices that contain $B_{r 1}\left(\theta_{k}, \phi_{k}\right)$ and $B_{r 2}\left(\theta_{k}, \phi_{k}\right)$, respectively, as well as their horizontal derivatives; $\mathbf{A}_{g}$ is a matrix derived from Eq. (4) or Eq. (8); $\alpha$ is a parameter that controls the weight of tangential geostrophy or tangential magnetostrophy; and $\boldsymbol{g}$ contains $\bar{U}_{l}^{m c}, \bar{U}_{l}^{m s}, \bar{W}_{l}^{m c}$, and $\bar{W}_{l}^{m s}$.

The number of unknowns for poloidal and toroidal scalars expanded into spherical harmonics is $2 L(L+2)=448$. The number of grid points on spherical surfaces at $r=r_{1}$ and $r=r_{2}$ is 45 in the $\theta$-direction and 90 in the $\phi$-direction. The linear Eq. (15) is solved using a Householder method. The parameter $\alpha$ is determined from a trade-off relationship between the tangentially geostrophic or tangentially magnetostrophic constraint and correlation of $\dot{B}_{r}^{\text {mod }}$ due to estimated flow to $\dot{B}_{r}^{\text {obs }}$ obtained from geomagnetic field data, or a relative misfit defined as

$$
M_{i}=\sqrt{\frac{\int\left(\dot{B}_{r i}^{\mathrm{mod}}-\dot{B}_{r i}^{\mathrm{obs}}\right)^{2} d S_{i}}{\int\left(\dot{B}_{r i}^{\mathrm{obs}}\right)^{2} d S_{i}}}
$$

where $\int d S_{i}$ is an integral over a spherical surface of radius $r=r_{i}$.

To solve the linear equation, two physical parameters, eddy kinematic viscosity and electrical conductivity of the Earth's core, must be given. Various values of eddy kinematic viscosity were proposed, e.g., $v_{\text {edd }} \sim 3 \mathrm{~m}^{2} \mathrm{~s}^{-1}$ (Braginsky 1991) and $v_{\text {edd }} \sim 7 \mathrm{~m}^{2} \mathrm{~s}^{-1}$ (Davis and Whaler 1997). Matsushima (2015) adopted their average, $v_{\text {edd }} \sim 5 \mathrm{~m}^{2} \mathrm{~s}^{-1}$, corresponding to an Ekman number, $E=v_{\text {edd }} / \Omega r_{0}^{2} \sim 6 \times 10^{-9}$. In this paper, the same value of eddy kinematic viscosity is used.

The thickness of the viscous boundary layer, $\delta_{E}$ or $\delta_{E H}$, contains $|\cos \theta|$ in the denominator. The value of $|\cos \theta|$ in the range between $\theta=5 \pi / 12$ and $\theta=7 \pi / 12$ is set at $|\cos (5 \pi / 12)|$ to avoid a singularity of $\delta_{E}$ at $\theta=\pi / 2$ (Matsushima 2015). Even for another range between $\theta=17 \pi / 36$ and $\theta=19 \pi / 36$, correlation coefficients between the resultant core flow model and the one by Matsushima (2015) are found to be more than 0.99. This indicates that the procedure to avoid a singularity at $\theta=\pi / 2$ does not have a severe influence on the core flow modeling. Thus, $\delta_{E} \sim 270-540 \mathrm{~m}$, $\xi_{1}=0.2 \mathrm{~km}<\delta_{\mathrm{E}}$, and $\xi_{2}=2 \mathrm{~km} \gg \delta_{\mathrm{E}}$ are adopted in this paper. As found from Eq. (9), $\delta_{E H}^{+} \sim 174-348 \mathrm{~m}$ even for $\Lambda=2$.

Regarding the other physical parameter, core electrical conductivity, Matsushima (2015) adopted $\sigma=3 \times 10^{5} \mathrm{~S} \mathrm{~m}^{-1} \quad$ (Stacey 1992). However, recent first-principles calculations and high-pressure high-temperature experiments suggest that core electrical conductivity can be higher than $\sigma=3 \times 10^{5} \mathrm{~S} \mathrm{~m}^{-1}$. For example, Pozzo et al. (2012) obtained $\sigma=1.11 \times 10^{6} \mathrm{~S} \mathrm{~m}^{-1}$ at the CMB from firstprinciple calculations. From high-pressure high-temperature experiments, Ohta et al. (2016) estimated $\sigma \sim 1 \times 10^{6} \mathrm{~S} \mathrm{~m}^{-1}$ for liquid $\mathrm{Fe}_{67.5} \mathrm{Ni}_{10} \mathrm{Si}_{22.5}$ at the CMB. It should be noted, however, that Konôpková et al. (2016) obtained a rather low value, $\sigma \sim 2.7 \times 10^{5} \mathrm{~S} \mathrm{~m}^{-1}$, from experiments. In this paper, core electrical conductivity, as a parameter, is investigated in the range from $\sigma=1 \times 10^{5} \mathrm{~S} \mathrm{~m}^{-1}$ to $\sigma=1 \times 10^{7} \mathrm{~S} \mathrm{~m}^{-1}$. 


\section{Results}

First, the effect of core electrical conductivity on tangentially geostrophic core flow below the viscous boundary layer at the $\mathrm{CMB}$ is investigated. Table 1 shows the mean velocity over spherical surfaces at $r=r_{1}$ and at $r=r_{2}$ for core electrical conductivity between $\sigma=1 \times 10^{5} \mathrm{~S} \mathrm{~m}^{-1}$ and $\sigma=1 \times 10^{7} \mathrm{~S} \mathrm{~m}^{-1}$. It is found that differences among mean velocity are $0.5 \%$ at most. Table 1 also lists correlation coefficients of $\nabla_{H} \cdot \boldsymbol{V}_{H i}$ and $\hat{\boldsymbol{r}} \cdot \nabla \times \boldsymbol{V}_{H i}$ for $\sigma=1 \times 10^{6} \mathrm{~S} \mathrm{~m}^{-1}$ and for another $\sigma$. The former and the latter correspond to the correlation coefficients for the poloidal and the toroidal components, respectively. It is found that the correlation coefficients are at least 0.98 . The core electrical conductivity is related to the diffusion term in the induction equation. That is, $\partial^{2} B_{r} / \partial r^{2}$ depends

Table 1 Mean velocity and correlation coefficients for the respective electrical conductivity values under the tangentially geostrophic constraint in $\mathbf{2 0 1 0}$

\begin{tabular}{llllll}
\hline $\boldsymbol{\sigma}\left[\mathbf{S ~ m}^{-1}\right]$ & $\mathbf{1} \times \mathbf{1 0}^{\mathbf{5}}$ & $\mathbf{3} \times \mathbf{1 0}^{\mathbf{5}}$ & $\mathbf{1} \times \mathbf{1 0}^{\mathbf{6}}$ & $\mathbf{3} \times \mathbf{1 0}^{\mathbf{6}}$ & $\mathbf{1 \times 1 \mathbf { 0 } ^ { \mathbf { 7 } }}$ \\
\hline$V_{1 \text { mean }}\left[\mathrm{km} \mathrm{yr}^{-1}\right]$ & 4.087 & 4.086 & 4.084 & 4.080 & 4.076 \\
$V_{2 \text { mean }}\left[\mathrm{km} \mathrm{yr}^{-1}\right]$ & 7.519 & 7.518 & 7.512 & 7.498 & 7.474 \\
$\nabla_{H} \cdot \boldsymbol{V}_{H 1}$ & 0.9999 & 0.9999 & - & 0.9996 & 0.9910 \\
$\nabla_{H} \cdot \boldsymbol{V}_{H 2}$ & 0.9998 & 0.9999 & - & 0.9990 & 0.9803 \\
$\hat{\boldsymbol{r}} \cdot \nabla \times \boldsymbol{V}_{H 1}$ & 0.9999 & 0.9999 & - & 0.9996 & 0.9924 \\
$\hat{\boldsymbol{r}} \cdot \nabla \times \boldsymbol{V}_{H 2}$ & 0.9999 & 0.9999 & - & 0.9995 & 0.9900 \\
\hline
\end{tabular}

Correlation coefficients of the horizontal divergence and the radial vorticity are computed between the one for $\sigma=1 \times 10^{6} \mathrm{~S} \mathrm{~m}^{-1}$ and that for another $\sigma$ on $\sigma$, and it is used to estimate $B_{r 1}$ and $B_{r 2}$ through the Taylor expansion. The result implies that core electrical conductivity has a limited effect on core flow models through the magnetic diffusion term under the tangentially geostrophic constraint.

It is worth noting, however, that the tangentially geostrophic constraint is known to be too strong, in particular, near the geographic equator; the $\theta$-component of core flow, $V_{\theta}$, at the equator must vanish under the constraint. Therefore, the geomagnetic secular variation around the equator is not well explained by such tangentially geostrophic flows (Wardinski et al. 2008). In other words, ageostrophic flows crossing the equator are necessary to explain the secular variation there. Such ageostrophic flows can be regarded as deviations from tangentially geostrophic flows, and they can be estimated by relaxing the tangentially geostrophic constraint (Pais et al. 2004; Asari and Lesur 2011). In their approach, a parameter, which corresponds to the controlling parameter, $\alpha$, in Eq. (15) in the present study, is changed so as to relax the constraint. The ageostrophic flows thus obtained are considered to result from the Lorentz force.

Hence, secondly, the effect of core electrical conductivity on tangentially magnetostrophic flow below the boundary layer is investigated. Under the tangentially magnetostrophic constraint, the core electrical conductivity is related not only to the magnetic diffusion, but also to the Lorentz force. Figure $1 \mathrm{a}-\mathrm{c}$ show fluid motions near the CMB at $r=r_{1}$ and $r=r_{2}$ for $\sigma=1 \times 10^{5} \mathrm{~S} \mathrm{~m}^{-1}$, $\sigma=1 \times 10^{6} \mathrm{~S} \mathrm{~m}^{-1}$, and $\sigma=1 \times 10^{7} \mathrm{~S} \mathrm{~m}^{-1}$, respectively,
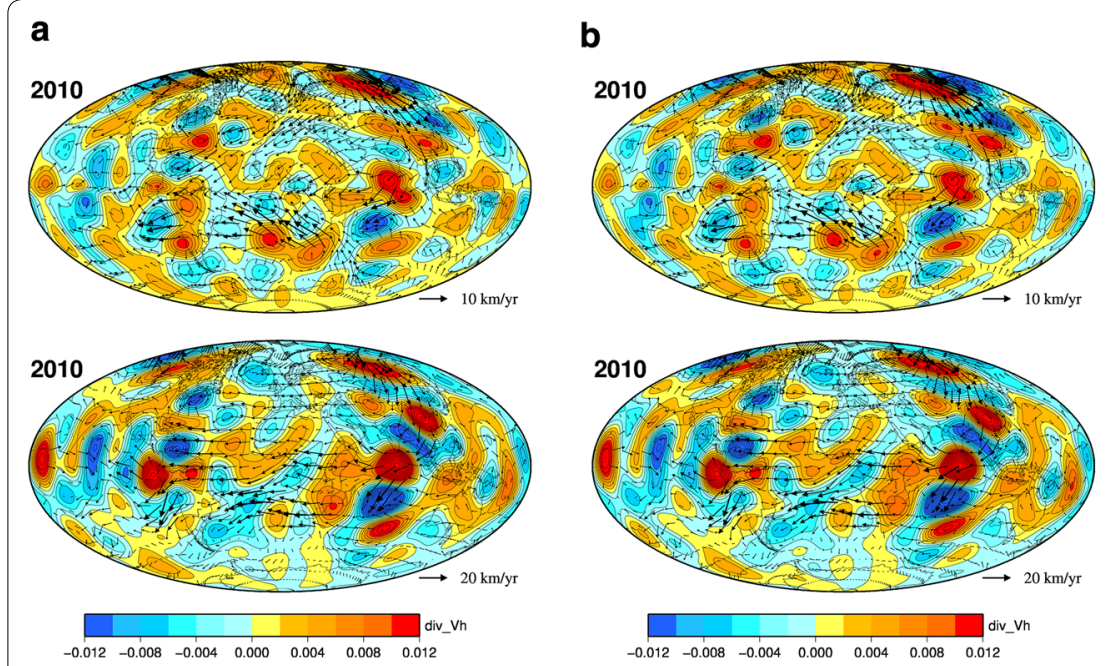

c
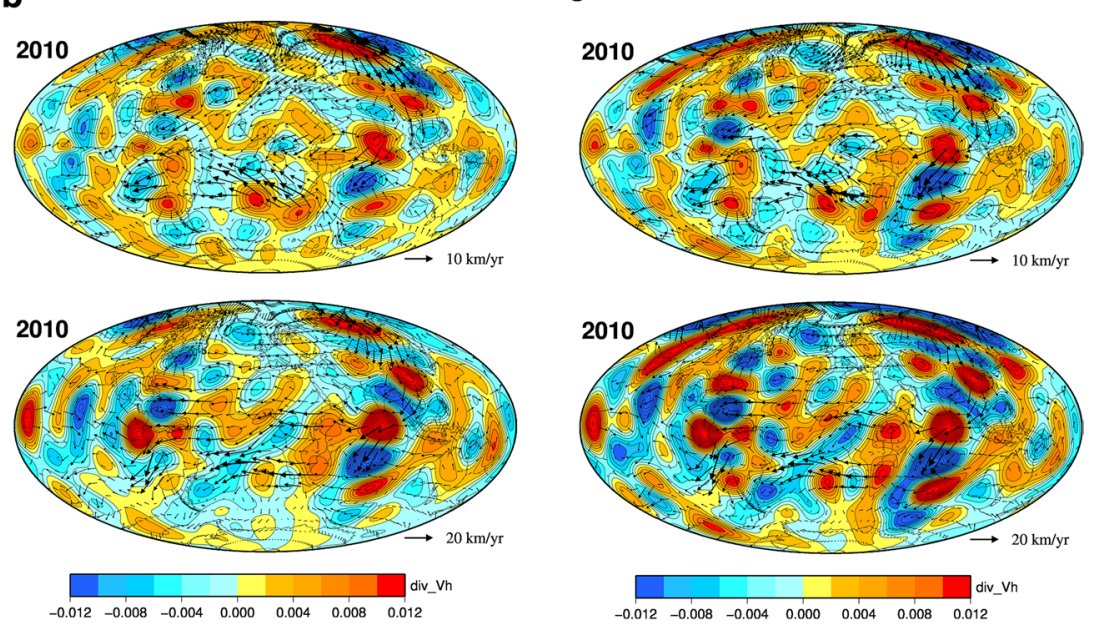

Fig. 1 Fluid motions near the core-mantle boundary under the tangentially magnetostrophic constraint. Upper and lower figures show fluid motions at $r=r_{1}$ and at $r=r_{2}$, respectively, for $\mathbf{a} \sigma=1 \times 10^{5} \mathrm{~S} \mathrm{~m}^{-1}, \mathbf{b} \sigma=1 \times 10^{6} \mathrm{~S} \mathrm{~m}^{-1}$, and $\mathbf{c} \sigma=1 \times 10^{7} \mathrm{~S} \mathrm{~m}^{-1}$ at the epoch of 2010 . Arrows show the horizontal flows, and color contours denote upwellings and downwellings given by $\nabla_{H} \cdot \boldsymbol{V}_{H}$. For $\rho=1.1 \times 10^{4} \mathrm{~kg} \mathrm{~m}^{-3}$, $\Omega=7.29 \times 10^{-5} \mathrm{rad} \mathrm{s}^{-1}$, and a root-mean-square value of the radial magnetic field at the CMB, $B_{\mathrm{r}} \approx 0.2 \mathrm{mT}, \Lambda=\sigma B_{r}^{2} / \rho \Omega \approx 0.005 \mathrm{for}$ $\sigma=10^{5} \mathrm{~S} \mathrm{~m}^{-1}, \Lambda \approx 0.05$ for $\sigma=10^{6} \mathrm{Sm}^{-1}$, and $\Lambda \approx 0.5$ for $\sigma=10^{7} \mathrm{~S} \mathrm{~m}^{-1}$ 
Table 2 Mean velocity and correlation coefficients for the respective electrical conductivity values under the tangentially magnetostrophic constraint in $\mathbf{2 0 1 0}$

\begin{tabular}{llllll}
\hline $\boldsymbol{\sigma}\left[\mathbf{S ~ m}^{-1}\right]$ & $\mathbf{1} \times \mathbf{1 0}^{\mathbf{5}}$ & $\mathbf{3} \times \mathbf{1 0 ^ { 5 }}$ & $\mathbf{1} \times \mathbf{1 0}^{\mathbf{6}}$ & $\mathbf{3} \times \mathbf{1 0}^{\mathbf{6}}$ & $\mathbf{1} \times \mathbf{1 0}^{\mathbf{7}}$ \\
\hline$V_{1}$ mean $\left[\mathrm{km} \mathrm{yr}^{-1}\right]$ & 4.131 & 4.145 & 4.114 & 3.892 & 4.342 \\
$V_{2 \text { mean }}\left[\mathrm{km} \mathrm{yr}^{-1}\right]$ & 7.680 & 7.710 & 7.612 & 7.604 & 7.319 \\
$\nabla_{H} \cdot \boldsymbol{V}_{H 1}$ & 0.9869 & 0.9923 & - & 0.9698 & 0.8756 \\
$\nabla_{H} \cdot \boldsymbol{V}_{H 2}$ & 0.9916 & 0.9944 & - & 0.9769 & 0.9229 \\
$\hat{\boldsymbol{r}} \cdot \nabla \times \boldsymbol{V}_{H 1}$ & 0.9738 & 0.9837 & - & 0.9625 & 0.8563 \\
$\hat{\boldsymbol{r}} \cdot \boldsymbol{\nabla} \times \boldsymbol{V}_{H 2}$ & 0.9845 & 0.9896 & - & 0.9765 & 0.8961 \\
\hline
\end{tabular}

Correlation coefficients of the horizontal divergence and the radial vorticity are computed between the one for $\sigma=1 \times 10^{6} \mathrm{~S} \mathrm{~m}^{-1}$ and that for another $\sigma$

at the epoch of 2010. Core flows for $\sigma=1 \times 10^{5} \mathrm{~S} \mathrm{~m}^{-1}$ are found to be similar to those for $\sigma=1 \times 10^{6} \mathrm{~S} \mathrm{~m}^{-1}$, whereas those for $\sigma=1 \times 10^{7} \mathrm{~S} \mathrm{~m}^{-1}$ are clearly different from those for $\sigma=1 \times 10^{6} \mathrm{~S} \mathrm{~m}^{-1}$. In fact, the twist of horizontal flows seen in an Ekman layer at $r=r_{1}$ and $r=r_{2}$ is similarly found in Fig. 1a and b, but horizontal flows at $r=r_{1}$ and $r=r_{2}$ in Fig. 1c for large $\Lambda$ are found to be much more parallel. The flow velocity averaged over spherical surfaces at $r=r_{2}$ decreases with an increase of $\sigma$, as listed in Table 2. It should be noted, however, that the horizontal divergence for $\sigma=1 \times 10^{7} \mathrm{~S} \mathrm{~m}^{-1}$ appears larger than that for $\sigma=1 \times 10^{6} \mathrm{~S} \mathrm{~m}^{-1}$. This dependence of poloidal and toroidal mean-flow magnitudes on the core electrical conductivity is obviously found in Fig. 2 . The mean velocity for the toroidal component does not increase or decrease with increasing core electrical conductivity. In contrast, the mean velocity for the poloidal component increases with increasing core electrical conductivity, as found from larger horizontal divergence for higher electrical conductivity. The reason why poloidal flow is larger for higher core electrical conductivity is likely to result from the Lorentz force on the tangentially magnetostrophic constraint, because tangentially geostrophic core flows are found not to be influenced by core electrical conductivity. The Coriolis force can be relatively unimportant for very high $\sigma$, as found in Eq. (8).

\section{Discussion}

To determine the cause, mean flow velocity is investigated under the tangentially geostrophic and tangentially magnetostrophic constraints. Equation (4) for the tangentially geostrophic flow can be given as

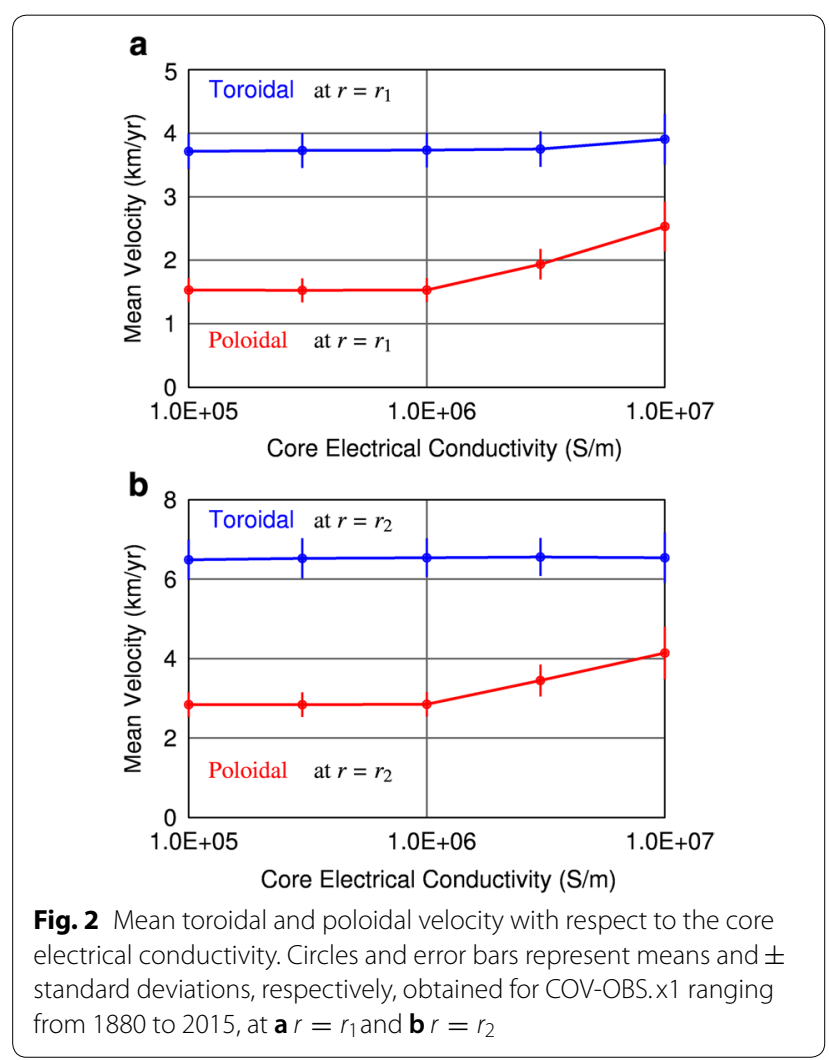

$$
\begin{aligned}
& \sum_{l=1}^{L} \sum_{m=0}^{l}\left[l(l+1) \cos \theta\left\{\bar{U}_{l}^{m c} \cos m \phi+\bar{U}_{l}^{m s} \sin m \phi\right\} P_{l}^{m}(\cos \theta)\right. \\
& \quad+\sin \theta\left\{\bar{U}_{l}^{m c} \cos m \phi+\bar{U}_{l}^{m s} \sin m \phi\right\} \frac{d P_{l}^{m}}{d \theta} \\
& \left.\quad+m\left\{-\bar{W}_{l}^{m c} \sin m \phi+\bar{W}_{l}^{m s} \cos m \phi\right\} P_{l}^{m}(\cos \theta)\right]=0 .
\end{aligned}
$$

The solutions of Eq. (17) are non-unique and underdetermined, although the number of unknowns is decreased as found in a basis for the tangentially geostrophic flow determined from the selection rule of the Gaunt integral (Le Mouël et al. 1985). Equation (17) has $m$-dependence in the $\phi$-direction as found from the selection rule, and it is possible to consider $\cos m \phi$ terms only from orthogonality of cosine and sine functions. Equation (17) can be reduced to

$\left\{l(l+1) \cos \theta P_{l}^{m}+\sin \theta \frac{d P_{l}^{m}}{d \theta}\right\} \bar{U}_{l}^{m c}+\left\{(l+2)(l+3) \cos \theta P_{l+2}^{m}+\sin \theta \frac{d P_{l+2}^{m}}{d \theta}\right\} \bar{U}_{l+2}^{m c}+m P_{l+1}^{m} \bar{W}_{l+1}^{m s}=0(l=m, m+2, m+4, \cdots)$ 
As mentioned above, the problem is underdetermined. In this study, therefore, a constraint

$$
\int\left\{\left(\bar{V}_{\theta 2}\right)^{2}+\left(\bar{V}_{\phi 2}\right)^{2}\right\} d S \rightarrow \min ,
$$

or

$$
\begin{aligned}
& \sum_{j=0}^{m+2 j+1 \leq L} \frac{(m+2 j)(m+2 j+1)}{2(m+2 j)+1}\left\{\left(\bar{U}_{m+2 j}^{m c}\right)^{2}\right. \\
& \left.+\left(\bar{W}_{m+2 j+1}^{m s}\right)^{2}\right\} \rightarrow \min
\end{aligned}
$$

is added, where $\int d S$ denotes a surface integral over a unit sphere. Hence, $\bar{U}_{l}^{m c}=1$ is given, and the other terms are computed as relative values by minimizing the following function, $\Psi_{g}$ :

$$
\Psi_{g}=\left[\nabla_{H} \cdot\left(\cos \theta \overline{\boldsymbol{V}}_{\mathrm{H}}\right)\right]^{2}+\alpha_{g} \int\left\{\left(\bar{V}_{\theta 2}\right)^{2}+\left(\bar{V}_{\phi 2}\right)^{2}\right\} d S,
$$

where $\alpha_{g}$ is a controlling parameter. Figure 3 shows the ratio of magnitude of mean toroidal flow to that of mean poloidal flow for $m=1$ to $m=6$ with respect to $\alpha_{g}$. The

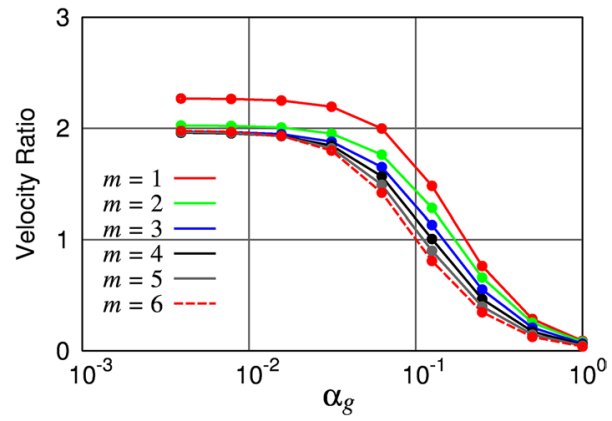

Fig. 3 Control parameter, $\alpha_{g}$, dependence of toroidal and poloidal mean flow ratios. Circles represent the ratio of the mean toroidal flow to the mean poloidal flow magnitudes at $r=r_{2}$ under the tangentially geostrophic constraint, for spherical harmonic order $m=1$ to $m=6$ truncation level for the spherical harmonics is increased from $L=14$ to $L=19$, to keep the number of toroidal components and poloidal components the same. The ratio of the magnitude of mean toroidal flow to that of mean poloidal flow is found to be approximately 2 . To confirm the effect of the truncation level, the ratio of the magnitude of mean toroidal flow to that of mean poloidal flow is computed for various values of $L$, as listed in Table 3. The result suggests that the effect of $L$ is not very significant.

Next, mean flow velocity under the tangentially magnetostrophic constraint is investigated. Using the same method as for the tangentially geostrophic flow, unknowns $\bar{U}_{l}^{m c}, \bar{U}_{l}^{m s}, \bar{W}_{l}^{m c}$, and $\bar{W}_{l}^{m s}$ relative to the provided $\bar{W}_{1}^{0}=1$ are computed by minimizing the function, $\Psi_{m}$ :

$$
\begin{aligned}
\Psi_{m}= & {\left[\nabla_{H} \cdot\left(2 \Omega \cos \theta \overline{\boldsymbol{V}}_{H}+\rho^{-1} \sigma B_{r 2}^{2} \overline{\boldsymbol{V}}_{H} \times \hat{\boldsymbol{r}}\right)\right]^{2} } \\
& +\alpha_{m} \int\left\{\left(\bar{V}_{\theta 2}\right)^{2}+\left(\bar{V}_{\phi 2}\right)^{2}\right\} d S,
\end{aligned}
$$

where $\alpha_{m}$ is a controlling parameter. Figure 4 shows the $\alpha_{m}$-dependence of the ratio of the magnitude of mean toroidal flow to that of mean poloidal flow in 2010 for $\sigma=10^{5} \mathrm{~S} \mathrm{~m}^{-1}, 10^{6} \mathrm{~S} \mathrm{~m}^{-1}$, and $10^{7} \mathrm{~S} \mathrm{~m}^{-1}$. The ratio

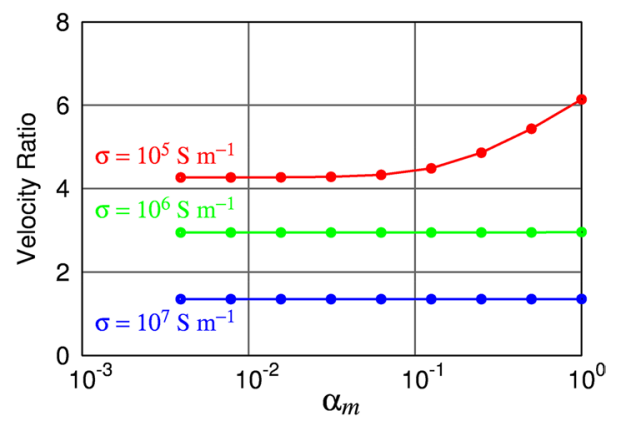

Fig. 4 Control parameter, $\alpha_{m}$, dependence of toroidal to poloidal

\begin{tabular}{|c|c|c|c|c|c|c|c|c|c|c|c|}
\hline \multicolumn{2}{|c|}{$m=1$} & \multicolumn{2}{|c|}{$m=2$} & \multicolumn{2}{|c|}{$m=3$} & \multicolumn{2}{|c|}{$m=4$} & \multicolumn{2}{|c|}{$m=5$} & \multicolumn{2}{|c|}{$m=6$} \\
\hline$L$ & Ratio & $L$ & Ratio & $L$ & Ratio & $L$ & Ratio & $L$ & Ratio & $L$ & Ratio \\
\hline 14 & 2.268 & 15 & 2.025 & 16 & 1.970 & 17 & 1.960 & 18 & 1.966 & 19 & 1.978 \\
\hline 24 & 2.268 & 25 & 2.025 & 26 & 1.969 & 27 & 1.959 & 28 & 1.963 & 29 & 1.973 \\
\hline 36 & 2.268 & 37 & 2.025 & 38 & 1.969 & 39 & 1.959 & 40 & 1.963 & 41 & 1.972 \\
\hline 48 & 2.268 & 49 & 2.025 & 50 & 1.969 & 51 & 1.959 & 52 & 1.963 & 53 & 1.972 \\
\hline
\end{tabular}
mean flow ratios. Circles represent the ratio of the mean toroidal to that of the mean poloidal flow magnitudes at $r=r_{2}$ under the tangentially magnetostrophic constraint, for $\sigma=1 \times 10^{5} \mathrm{~S} \mathrm{~m}^{-1}$, $\sigma=1 \times 10^{6} \mathrm{~S} \mathrm{~m}^{-1}$, and $\sigma=1 \times 10^{7} \mathrm{~S} \mathrm{~m}^{-1}$ at the epoch of 2010

Table 3 Mean toroidal flow to poloidal flow ratio for different truncation levels of spherical harmonics 
clearly decreases with increasing $\sigma$. It is likely that this result arises from the effect of the Lorentz force proportional to $\sigma$. This tendency is invariable for other epochs. It should be noted that these ratios are not necessarily equal to those obtained from a core flow model, simply because core flows are derived from geomagnetic field data including secular variations under the tangentially magnetostrophic constraint.

Asari and Lesur (2011) attempted to compare the tangentially geostrophic and the tangentially magnetostrophic constraints by examining the resolution matrix. They found that the tangentially geostrophic constraint mainly influences the poloidal flow. On the other hand, they pointed out that the tangentially magnetostrophic constraint rather mitigates the poloidal flow. This may be related with the present result that the mean poloidal flow magnitude increases with increasing core electrical conductivity.

Driscoll and $\mathrm{Du}$ (2019) derived a phase diagram of the dynamo regime determined from core electrical conductivity and temperature. To maintain dynamo action driven by thermal convection, higher heat flux at the $\mathrm{CMB}$ is required for higher electrical conductivity, which implies higher thermal conductivity. The core electrical conductivity is likely to have been increasing, as the core temperature has been decreasing. This suggests that a thermally driven dynamo was converted into a compositionally driven dynamo by way of a thermally and compositionally driven dynamo. A similar transition of core dynamics due to the thermal history of the Earth is discussed later.

To this point, focus has been on core electrical conductivity for investigation of core flow estimation. However, as demonstrated in Eq. (8), the tangentially magnetostrophic constraint does not depend on core electrical conductivity alone. The relative importance of the Lorentz force to the Coriolis force can be measured by the Elsasser number, $\Lambda$, as given by Eq. (10). The Elsasser number employed in the present study can be defined as the traditional form appropriate for steady imposed magnetic fields. This was shown by Soderlund et al. (2012, 2015), who found that the dynamic Elsasser number, $\Lambda_{d}$, represents the ratio of the Lorentz to Coriolis forces better than the traditional one. However, it should be indicated that $\Lambda_{d}$ is obtained from the magnetic field strength averaged over the core. In contrast, $\Lambda$, as used in this paper, is locally defined at respective points on a spherical surface. In this sense, the traditional form of the Elsasser number is likely to be more appropriate for use in this study.

It is known that the rotation rate of the Earth has been decreasing due to tidal friction with the Moon. It is currently approximately $24 \mathrm{~h}$, but it could have been as little as $4-6 \mathrm{~h}$ immediately after the Moon formed (e.g.,
Goldreich 1966; Mignard 1982). It follows that $\Omega$ was approximately six to four times larger than the present value. Thus, the denominator of $\Lambda=\sigma B_{r}^{2} / \rho \Omega|\cos \theta|$ is likely to have been decreasing throughout the history of the Earth. However, the core temperature has been decreasing since the formation of the core, as found from the thermal evolution of the Earth. This suggests that the core electrical conductivity in the past could have been smaller than the present value, and that the numerator of $\Lambda$ has been increasing. These circumstances indicate that the Elsasser number, $\Lambda$, could have been smaller in the past than at present. That is, core flow in the past could have been more geostrophic than the present flow state. With time, the rotation rate of the Earth decreases, and the core electrical conductivity increases. Hence, the Elsasser number, $\Lambda$, will be increasing. This implies that the style of magnetic field generation by poloidal and toroidal motions in the core has been changing.

In the present study, it is implicitly assumed that convective motions occur in the entire outer core. Alternatively, presence of a thermally or compositionally stably stratified layer at the top of the outer core is suggested by some seismic studies (e.g., Tanaka 2007; Helffrich and Kaneshima 2010), material studies (e.g., Pozzo et al. 2012; Gomi et al. 2013), and geomagnetic studies (e.g., Whaler 1980; Buffett 2014). If it is the case, the core surface flow should be purely toroidal without upwelling or downwelling. This point was examined by Whaler (1980), whose conclusion supported stable stratification at the core surface. However, this result depends on the validity of frozen-flux hypothesis, as Gubbins (2007) pointed out a possible effect of magnetic diffusion on geomagnetic secular variations. Asari and Lesur (2011) found that the purely toroidal flow is incompatible with the tangentially magnetostrophic flow. Then, Lesur et al. (2015) concluded that the purely toroidal flow at the core surface cannot explain the observed geomagnetic field, although a small poloidal flow or magnetic diffusion may compensate for an incompatible part of geomagnetic secular variations. Furthermore, Takehiro and Lister (2001) demonstrated that a stably stratified layer at the top of the core can be penetrated by columnar convection depending on the rotation rate of the Earth and the horizontal scale of vortices. Thus, even if a stably stratified layer is present at the top of the outer core, it is impossible to ignore the presence of poloidal motion near the core surface. Further discussion on this point is beyond the scope of the present study, and the related problems should be addressed in the future. 


\section{Conclusions}

In this paper, the effect of core electrical conductivity in the range between $10^{5} \mathrm{~S} \mathrm{~m}^{-1}$ and $10^{7} \mathrm{~S} \mathrm{~m}^{-1}$ on core surface flow models was investigated. Core electrical conductivity is related to two terms in the equation to be solved: the magnetic diffusion term in the induction equation and the Lorentz force term in the Navier-Stokes equation. Tangentially geostrophic and tangentially magnetostrophic constraints were imposed for core flow beneath the viscous boundary layer at the core-mantle boundary to derive a core surface flow model from a geomagnetic field model. Magnetic diffusivity is inversely proportional to electrical conductivity, whereas the Lorentz force term is proportional to electrical conductivity. Under the tangentially geostrophic constraint, only the magnetic diffusion term has any effect on core surface flow models. It was found that core electrical conductivity has a limited effect on core flow models. In contrast, under the tangentially magnetostrophic constraint, it was found that the mean poloidal flow increases with an increase of core electrical conductivity (Fig. 2). This result arises from the Lorentz force, as found from Figs. 3 and 4, where the ratio of the magnitude of mean toroidal flow to that of mean poloidal flow is shown with respect to control parameters under the tangentially geostrophic and tangentially magnetostrophic constraints, respectively.

Furthermore, this result suggests that the ratio of the magnitude of mean toroidal flow to that of mean poloidal flow has been changing with secular change of the Elsasser number given by the ratio of the Lorentz and Coriolis forces. The Elsasser number has been increasing throughout the evolution of the Earth, because the rotation rate of the Earth has been decreasing and the core electrical conductivity has been increasing due to the decrease in core temperature. If the ratio can be estimated from magnetic field measurements of a planet, it may provide information on the core electrical conductivity of the planet.

\section{Abbreviation}

CMB: Core-mantle boundary.

\section{Acknowledgements}

The author is very grateful to the two anonymous reviewers for their useful and constructive comments. The author thanks Editage (https://www.edita ge.com) for English language editing of the original manuscript.

\section{Authors' contributions}

MM carried out everything related to this manuscript. The author read and approved the final manuscript.

\section{Funding}

This study was supported by JSPS KAKENHI Grant Numbers JP16H01116 and $15 \mathrm{H} 05832$
Availability of data and materials

The results of core surface flow are available from the author (masaki.matsushima@eps.sci.titech.ac.jp). A geomagnetic field model, COV-OBS.x1, is available from https://www.spacecenter.dk/files/magnetic-models/COV-OBSx1/

Ethics approval and consent to participate

Not applicable.

Consent for publication

Not applicable.

\section{Competing interests}

The author declares that he has no competing interests.

\section{Appendix: Expression of horizontal flow in an Ekman-Hartmann boundary layer}

The Elsasser number in this study is defined as $\Lambda=\sigma B_{r}^{2} / \rho \Omega$ (without $\theta$-dependence). According to Soderlund et al. (2012), this is a traditional form denoted by $\Lambda_{i}$, being the ratio of the Lorentz force $\rho^{-1} \boldsymbol{J} \times \boldsymbol{B}$ to the Coriolis force $\boldsymbol{\Omega} \times \boldsymbol{V}$ with $\boldsymbol{J}=\sigma(\boldsymbol{E}+\boldsymbol{V} \times \boldsymbol{B}) \approx \sigma \boldsymbol{V} \times \boldsymbol{B}$ as

$$
\Lambda_{i}=\frac{J B}{\rho \Omega V}=\frac{\sigma V B^{2}}{\rho \Omega V}=\frac{\sigma B^{2}}{\rho \Omega} .
$$

Alternatively, Soderlund et al. (2012) defined the dynamic Elsasser number, $\Lambda_{d}$ with $\boldsymbol{J}=\mu_{0}^{-1} \nabla \times \boldsymbol{B}$ as

$$
\Lambda_{d}=\frac{J B}{\rho \Omega V}=\frac{B^{2}}{\rho \mu_{0} \Omega V \ell_{B}},
$$

where the electric current density is estimated as $J \sim B / \mu_{0} \ell_{B}$. Soderlund et al. (2012) pointed out that $\Lambda_{d}$ represents the ratio of the Lorentz force to the Coriolis force better than the traditional one. $\boldsymbol{E}=\mathbf{0}$, as assumed above, means that the magnetic field is not strongly time variant. This corresponds to a magnetoconvective system, in which a magnetic field is imposed.

Temporal variation of the radial magnetic field, $\dot{B}_{r}$, is generated by the interaction between $V_{H}$ and imposed $B_{r}$. Therefore, $\Lambda_{i}$ corresponding to $\boldsymbol{J} \approx \sigma \boldsymbol{V} \times \boldsymbol{B}$ is appropriate. Then, the horizontal component of current density, which is much larger than the radial component, $J_{r} \ll\left|J_{H}\right|$, is given as

$$
\boldsymbol{J}_{H} \approx \sigma(\boldsymbol{V} \times \boldsymbol{B})_{H} \approx \sigma B_{r} \boldsymbol{V}_{H} \times \hat{\boldsymbol{r}} .
$$

Next, using Eq. (24), I derive the expression of horizontal flow in an Ekaman-Hartmann boundary layer (e.g., Busse et al. 2007). The equation of motion including the effect of Earth's rotation and magnetic field, neglecting the inertia term, can be given as

$$
-\frac{1}{\rho} \nabla p-2 \boldsymbol{\Omega} \times \boldsymbol{V}+\frac{A}{\rho} \hat{\boldsymbol{r}}+\frac{1}{\rho} \boldsymbol{J} \times \boldsymbol{B}+v \nabla^{2} \boldsymbol{V}=\mathbf{0} .
$$

The horizontal components of Eq. (25) can be written as 


$$
\begin{aligned}
& -\frac{1}{\rho} \frac{1}{r} \frac{\partial p}{\partial \theta}+2 \Omega \cos \theta V_{\phi}+\frac{1}{\rho} J_{\phi} B_{r}+v \frac{\partial^{2} V_{\theta}}{\partial r^{2}} \approx 0, \\
& -\frac{1}{\rho} \frac{1}{r \sin \theta} \frac{\partial p}{\partial \phi}-2 \Omega \cos \theta V_{\theta}-\frac{1}{\rho} J_{\theta} B_{r}+v \frac{\partial^{2} V_{\phi}}{\partial r^{2}} \approx 0,
\end{aligned}
$$

where $\nabla^{2} \approx \partial^{2} / \partial r^{2}$ is presumed. Using Eq. (24), one can obtain the magnetostrophic part of Eqs. (26a) and (26b) as

$$
\begin{aligned}
& -\frac{1}{\rho} \frac{1}{r} \frac{\partial p}{\partial \theta}+2 \Omega \cos \theta \bar{V}_{\phi}-\frac{\sigma}{\rho} B_{r}^{2} \bar{V}_{\theta}=0, \\
& -\frac{1}{\rho} \frac{1}{r \sin \theta} \frac{\partial p}{\partial \phi}-2 \Omega \cos \theta \bar{V}_{\theta}-\frac{\sigma}{\rho} B_{r}^{2} \bar{V}_{\phi}=0 .
\end{aligned}
$$

Taking

$$
V_{\theta}=\bar{V}_{\theta}+v_{\theta}, V_{\phi}=\bar{V}_{\phi}+v_{\phi}, \frac{\partial^{2} \bar{V}_{\theta}}{\partial r^{2}}=\frac{\partial^{2} \bar{V}_{\phi}}{\partial r^{2}}=0,
$$

one can obtain

$$
\begin{aligned}
& 2 \Omega \cos \theta v_{\phi}-\frac{\sigma}{\rho} B_{r}^{2} v_{\theta}+\frac{\partial^{2} v_{\theta}}{\partial r^{2}}=0, \\
& -2 \Omega \cos \theta v_{\theta}-\frac{\sigma}{\rho} B_{r}^{2} v_{\phi}+\frac{\partial^{2} v_{\phi}}{\partial r^{2}}=0 .
\end{aligned}
$$

Solving these equations, one obtains a solution in terms of linear combination as

$$
\begin{aligned}
v_{\theta}= & c_{1} e^{\zeta \gamma e^{i \beta}}+c_{2} e^{-\zeta \gamma e^{i \beta}}+c_{3} e^{\zeta \gamma e^{-i \beta}}+c_{4} e^{-\zeta \gamma e^{-i \beta}} \\
v_{\phi}= & (\operatorname{sgn} \cos \theta)\left(-i c_{1} e^{\zeta \gamma e^{i \beta}}-i c_{2} e^{-\zeta \gamma e^{i \beta}}\right. \\
& \left.+i c_{3} e^{\zeta \gamma e^{-i \beta}}+i c_{4} e^{-\zeta \gamma e^{-i \beta}}\right)
\end{aligned}
$$

where $\zeta=\sqrt{2} \xi / \delta_{E}, \quad \xi=r_{0}-r, \quad \gamma=\left(1+\Lambda^{4} / 4\right)^{1 / 4}$, $\cos 2 \beta=\Lambda / 2 \gamma^{2}$, sin $2 \beta=1 / \gamma^{2}$, and $c_{1}, c_{2}, c_{3}, c_{4}$ are constants. $v_{\theta}$ and $v_{\phi}$ must be finite for $\zeta \rightarrow \infty$, so that $c_{1}=c_{3}=0$, and $v_{\theta}$ and $v_{\phi}$ must vanish at $\zeta=0$, so that $v_{\theta}=-\bar{V}_{\theta}$ and $v_{\phi}=-\bar{V}_{\phi}$. Then, one can obtain the solution as

$$
\begin{aligned}
V_{\theta}= & \bar{V}_{\theta}\left\{1-e^{-\zeta \gamma \cos \beta} \cos (\zeta \gamma \sin \beta)\right\} \\
& -(\operatorname{sgn} \cos \theta) \bar{V}_{\phi} e^{-\zeta \gamma \cos \beta} \sin (\zeta \gamma \sin \beta), \\
V_{\phi}= & \bar{V}_{\phi}\left\{1-e^{-\zeta \gamma \cos \beta} \cos (\zeta \gamma \sin \beta)\right\} \\
& +(\operatorname{sgn} \cos \theta) \bar{V}_{\theta} e^{-\zeta \gamma \cos \beta} \sin (\zeta \gamma \sin \beta) .
\end{aligned}
$$

Using

$$
\cos \beta=\sqrt{\gamma^{2}+\Lambda / 2} / \sqrt{2} \gamma
$$

and $\sin \beta=\sqrt{\gamma^{2}-\Lambda / 2} / \sqrt{2} \gamma$, one can obtain

$$
\zeta \gamma \cos \beta=\frac{\xi}{\delta_{E H}^{+}} \text {with } \delta_{E H}^{+}=\frac{\delta_{E}}{\left\{\left(1+\Lambda^{2} / 4\right)^{1 / 2}+\Lambda / 2\right\}^{1 / 2}},
$$

$$
\zeta \gamma \sin \beta=\frac{\xi}{\delta_{E H}^{-}} \text {with } \delta_{E H}^{-}=\frac{\delta_{E}}{\left\{\left(1+\Lambda^{2} / 4\right)^{1 / 2}-\Lambda / 2\right\}^{1 / 2}}
$$

Hence,

$$
\begin{aligned}
\boldsymbol{V}_{H}= & \overline{\boldsymbol{V}}_{H}\left\{1-\exp \left(-\frac{\xi}{\delta_{E H}^{+}}\right) \cos \left(\frac{\xi}{\delta_{E H}^{-}}\right)\right\} \\
& +(\operatorname{sgn} \cos \theta) \hat{\boldsymbol{r}} \times \overline{\boldsymbol{V}}_{H} \exp \left(-\frac{\xi}{\delta_{E H}^{+}}\right) \sin \left(\frac{\xi}{\delta_{E H}^{-}}\right) .
\end{aligned}
$$

Received: 9 March 2020 Accepted: 4 September 2020 Published online: 26 November 2020

\section{References}

Asari S, Lesur V (2011) Radial vorticity constraint in core flow modeling. J Geophys Res 116:B11101. https://doi.org/10.1029/2011JB008267 Benton ER, Muth LA (1979) On the strength of electric currents and zonal magnetic fields at the top of the Earth's core: Methodology and preliminary estimates. Phys Earth Planet Int 20:127-133

Braginsky SI (1991) Towards a realistic theory of the geodynamo. Geophys Astrophys Fluid Dyn 60:89-134

Busse F, Dormy E, Simitev R, Soward A (2007) Dynamics of rotating fluids. In: Dormy E, Soward AM (eds) Mathematical Aspects of Natural Dynamos. CRC Press, New York

Buffett B (2014) Geomagnetic fluctuations reveal stable stratification at the top of the Earth's core. Nature 507:484-487

Christensen UR, Wicht J (2015) Numerical dynamo simulation. In: Olson P (ed) Treatise on Geophysics, 2nd edn, vol 8, Elsevier, Amsterdam

Chulliat A, Olsen N (2010) Observation of magnetic diffusion in the Earth's outer core from Magsat, Ørsted, and CHAMP data. J Geophys Res 115:2009JB006994

Davis RG, Whaler KA (1997) The 1969 geomagnetic impulse and spin-up of Earth's liquid core. Phys Earth Planet Inter 103:181-194

Driscoll PE, Du Z (2019) Geodynamo conductivity limits. Geophys Res Lett 46:7982-7989. https://doi.org/10.1029/2019GL082915

Gillet N, Barrois O, Finlay CC (2015) Stochastic forecasting of the geomagnetic field from the COV-OBS.x1 geomagnetic field model, and candidate models for IGRF-12. Earth Planets Space 67:71. https://doi. org/10.1180/s40623-015-0225-z

Goldreich P (1966) History of the lunar orbit. Rev Geophys 4:411-439

Gomi H, Ohta K, Hirose K, Labrosse S, Caracas R, Verstraete MJ, Hernlund JW (2013) The high conductivity of iron and thermal evolution of the Earth's core. Phys Earth Planet Inter 224:88-103

Gubbins D (2007) Geomagnetic constraints on stratification at the top of Earth's core. Earth Planets Space 59:661-664

Helffrich G, Kaneshima S (2010) Outer-core compositional stratification from observed core wave speed profiles. Nature 468:807-812

Holme R (2015) Large-scale flow in the core. In: Olson P (ed) Treatise on Geophysics, 2nd edn, vol 8, Elsevier, Amsterdam

Kageyama A, Sato T (1997) Generation mechanism of a dipole field by a magnetohydrodynamic dynamo. Phys Rev E 55:4617-4626 
Konôpková Z, McWilliams RS, Gómez-Pérez N, Goncharov AF (2016) Direct measurement of thermal conductivity in solid iron at planetary core conditions. Nature 534:99-101

Le Mouël JL, Gire C, Madden T (1985) Motions at the core surface in geostrophic approximation. Phys Earth Planet Int 39:270-287

Lesur V, Whaler K, Wardinski I (2015) Are geomagnetic data consistent with stably stratified flow at the core-mantle boundary? Geophys J Int 201(2):929-946

Matsushima M (2015) Core surface flow modelling with geomagnetic diffusion in a boundary layer. Geophys J Int 202:1495-1504. https://doi. org/10.1093/gji/ggv233

Metman MC, Livermore PW, Mound JE (2018) The reversed and normal flux contribution to axial dipole decay for 1880-2015. Phys Earth Planet Int 276:106-117. https://doi.org/10.1016/j.pepi.2017.06.007

Mignard F (1982) Long time integration of the Moon's orbit. In: Brosche P, Sündermann J (eds) Tidal friction and the Earth's rotation II. Springer, Berlin

Ohta K, Kuwayama Y, Hirose K, Shimizu K, Ohishi Y (2016) Experimental determination of the electrical resistivity of iron at Earth's core conditions. Nature 534:95-98

Olson P, Christensen U, Glatzmaier GA (1999) Numerical modeling of the geodynamo: Mechanics of field generation and equilibration. J Geophys Res 104:10383-10404

Pais MA, Oliveira O, Nogueira F (2004) Nonuniqueness of inverted core-mantle boundary flows and deviations from tangential geostrophy. J Geophys Res 109:B08103. https://doi.org/10.1029/2004JB003012

Pedlosky J (1987) Geophysical Fluid Dynamics, 2nd edn. Springer, Berlin

Pozzo M, Davies C, Gubbins D, Alfè D (2012) Thermal and electrical conductivity of iron at Earth's core conditions. Nature 485:355-358

Roberts PH, Scott S (1965) On analysis of the secular variations, 1: A hydromag netic constraint: theory. J Geomag Geoelectr 17:137-151

Shimizu H (2006) On the use of boundary layer compatibility conditions for geodynamo modeling. E221-P001, Japan Geoscience Union Meeting 2006
Soderlund KM, King EM, Aurnou JM (2012) The influence of magnetic fields in planetary dynamo models. Earth Planet Sci Lett 333-334:9-20. https:// doi.org/10.1016/j.epsl.2012.03.038

Soderlund KM, Sheyko A, King EM, Aurnou JM (2015) The competition between Lorentz and Coriolis forces in planetary dynamos. Prog Earth Planet Sci 2:24. https://doi.org/10.1186/s40645-015-0054-5

Stacey FD (1992) Physics of the Earth, 3rd edn. Bookfield Press, Australia, p 513

Takahashi F, Katayama JS, Matsushima M, Honkura Y (2001) Effects of boundary layers on magnetic field behavior in an MHD dynamo model. Phys Earth Planet Inter 128:149-161

Takehiro S, Lister JR (2001) Penetration of columnar convection into an outer stably stratified layer in rapidly rotating spherical fluid shells. Earth Planet Sci Lett 187(3-4):357-366

Tanaka S (2007) Possibility of a low P-wave velocity layer in the outermost core from global SmKS waveforms. Earth Planet Sci Lett 259(3-4):486-499

Tarduno JA, Cottrell RD, Watkeys MK, Hofmann A, Doubrovine PV, Mamajek EE, Liu D, Sibeck DG, Neukirch LP, Usui T (2010) Geodynamo, solar wind, and magnetopause 3.4 to 3.45 billion years ago. Science 327:1238-1240

Wardinski I, Holme R, Asari S, Mandea M (2008) The 2003 geomagnetic jerk and its relation to the core surface flows. Earth Planet Sci Lett 267:468-481

Whaler K (1980) Does the whole of the Earth's core convect? Nature 287(5782):528-530

Xu J, Zhang P, Haule K, Minar J, Wimmer S, Ebert H, Cohen RE (2018) Thermal conductivity and electrical resistivity of solid iron at Earth's core conditions from first principles. Phys Rev Lett 121:096601. https://doi. org/10.1103/PhysRevLett.121.096601

\section{Publisher's Note}

Springer Nature remains neutral with regard to jurisdictional claims in published maps and institutional affiliations.

\section{Submit your manuscript to a SpringerOpen ${ }^{\circ}$ journal and benefit from:}

- Convenient online submission

- Rigorous peer review

- Open access: articles freely available online

- High visibility within the field

- Retaining the copyright to your article

Submit your next manuscript at $\gg$ springeropen.com 